\title{
Impact of chest subcutaneous fat on the occurrence of central venous port-related infectious complications in cancer patients
}

\author{
Jumpei Shibata ${ }^{1}$ (D) Hidetaka Kawamura ${ }^{2} \cdot$ Kazuhiro Hiramatsu $^{1}$ (i) $\cdot$ Michitaka Honda $^{2} \cdot$ Yoshihisa Shibata $^{1}$. \\ Taro Aoba $^{1}$ - Masahiro Fujii ${ }^{1}$ - Atsuki Arimoto ${ }^{1}$ - Akira Ito ${ }^{1} \cdot$ Kenta Ishii $^{1}$ - Kojiro Omiya ${ }^{1}$ - Mariko Asai ${ }^{1}$. \\ Takuya Arakawa $^{1}$ • Hirotake Gonda ${ }^{1}$. Shuhei Asai ${ }^{1} \cdot$ Takuya Hasegawa $^{1} \cdot$ Kento Kawashima $^{1} \cdot$ Takehito Kato $^{1}$ (D)
}

Received: 9 July 2020 / Accepted: 24 February 2021 / Published online: 10 March 2021

(C) The Author(s) 2021

\begin{abstract}
Purpose There is no concrete evidence to support the association between the amount of subcutaneous fat area (SFA) in the central venous port-insertion site (precordium) and port-related complications. We aimed to investigate the relationship between SFA in the midclavicular line and postoperative infectious complications in patients undergoing port-insertion surgery.

Methods This was a single-institute and historical cohort study of 174 patients who underwent first central venous port implantation surgery for chemotherapy between January 2014 and December 2018. SFA in the midclavicular line was measured using preoperative computed tomography scans. The patients were divided into three groups according to SFA amount tertiles, and we investigated the association of SFA with infectious and all-cause complication events within 1 year.

Results Within a median follow-up of 306 days, the patients with intermediate SFA had significantly higher infection-free survival than those with low and high SFA (low vs. intermediate vs. high: $80.4 \%$ vs. $97.7 \%$ vs. $83.4 \%$, respectively, $p=0.034$ ). In contrast, there was no significant difference in the overall complication-free survival among the groups (low vs. intermediate vs. high: $80.4 \%$ vs. $88.9 \%$ vs. $81.8 \%$, respectively, $p=0.29$ ). Low SFA was independently associated with high risk of infectious complications (hazard ratio, 9.45; 95\% confidence interval, 1.07-83.22, $p=0.043$ ).

Conclusion Low SFA in the midclavicular line was an independent risk factor for infectious complications in the chemotherapy setting. This practical indicator can be useful for optimizing patients' nutritional status and when considering other types of vascular access to support administration of intravenous chemotherapy.
\end{abstract}

Keywords Computed tomography $\cdot$ Subcutaneous fat area $\cdot$ Implantable central venous port · Infectious complication

\section{Introduction}

The central venous (CV) port system has become an essential device that facilitates long-term administration of chemotherapy. The CV port is completely implantable and enables the administration of various anticancer agents with lower risk of extravasation. Thus, patients report higher satisfaction and

Jumpei Shibata

shibata-junpei@toyohashi-mh.jp

1 Department of General Surgery, Toyohashi Municipal Hospital, 50 Aza Hachiken Nishi, Aotake-Cho, Toyohashi, Aichi 441-8570, Japan

2 Department of Minimally Invasive Surgical and Medical Oncology, Fukushima Medical University, 7-115 Yatsuyamada, Koriyama, Fukushima 963-8563, Japan lower anxiety [1]. However, despite their apparent benefits, $\mathrm{CV}$ ports can also be a source of cancer treatment-related morbidity, such as port-related infections [2]. Cancer patients who undergo chemotherapy are at a particularly higher risk of CV port-related infectious complications owing to their inherent cancer-related characteristics, including malnutrition and immune deficiency. CV port-related infectious complications can delay chemotherapy and indirectly lead to disease progression. Thus, identifying risk factors for $\mathrm{CV}$ port-related infectious complications is important in the management of cancer patients.

Cancer patients with malnutrition can be susceptible to $\mathrm{CV}$ port-related infectious complications because of their little subcutaneous fat in the surgical site, which may induce dermal necrosis and eventual exposure of the $\mathrm{CV}$ port. The close distance between the device and the outer skin may contribute to easy invasion of pathogens. Although surgeons believe that 
low subcutaneous fat in the port-insertion site might be a potential risk factor for port-related infectious complications, no study has reported an association between the subcutaneous fat area (SFA) and port-related infection.

Generally, body mass index (BMI) is used as an indicator of nutritional status and an index of obesity. However, the BMI does not specifically reflect the actual body's composition of muscle, visceral adipose, and subcutaneous adipose tissues [3]. In comparison, computed tomography (CT)-derived analytic imaging of body composition may lead to a more specific assessment of infection risk at the operative site [4]. More specifically, in $\mathrm{CV}$ port insertion, this objective measurement may potentially reflect the surgeons' preoperative subjective assessment of the operative site of the SFA in the midclavicular line. Within this context, we speculate that applying these objective surgical site assessment measures might inform preoperative risk evaluation and contribute to clinical decision-making.

We aimed to evaluate the association between subcutaneous fat in the midclavicular line and port-related infectious complications in adult patients undergoing CV port insertion for chemotherapy. We hypothesized that low SFA would be an independent risk factor for $\mathrm{CV}$ port-related infectious complications.

\section{Patients and methods}

\section{Study design and participants}

This was a single-center, historical cohort study of patients who underwent their first $\mathrm{CV}$ port implantation surgery between January 2014 and December 2018. The exclusion criteria were age under 20 years, CV port implantation for non-chemotherapy purposes, no CT scan within 90 days before $\mathrm{CV}$ port implantation, missing data, and multiple $\mathrm{CV}$ port insertions during the study period. We excluded minors younger than 20 years since we sought a study population of adults with similar cancers and treatment regimens. We defined the age of 65 years as the cut-off, since persons over that age are categorized as "elderly" in our country. Figure 1 shows the patient enrollment flowchart.

This study was approved by the Institutional Review Board of Toyohashi Municipal Hospital and was conducted in accordance with the ethical standards of the respective committees on human experimentation (institutional and national) and the 1964 Helsinki Declaration and its later amendments. Consent to participate in the study was obtained using an optout method.

\section{Measurement and assessment of SFA at the midclavicular line}

Patients underwent CT within 90 days before the procedure using a 64-row multi-detector scanner (Aquilion 64; Canon
Medical Systems, Tochigi, Japan) with them positioned in the decubitus dorsalis position. The reason for defining 90 days as the maximum time window for the pre-procedural CT scan is that the judgment regarding response or progression of cancer is usually performed within 90 days. The images obtained were transferred to a workstation (SYNAPSE VINCENT; FUJIFILM, Tokyo, Japan), wherein SFA at the level of the umbilicus was utilized to measure the midclavicular level semi-automatically (Fig. 2). The subsequent calculation process was as follows: first, the researcher manually adjusted the height of the axial CT scan at the midclavicular line. Second, the workstation semi-automatically detected and calculated the SFA according to the Hounsfield scale $(-50$ to -100 Hounsfield units). Third, the researcher readjusted the area manually if the semi-automatically detected area was obviously wrong.

The patients were divided into three groups, low, intermediate, and high, according to the SFA amount tertiles in the midclavicular line.

\section{Data collection and outcomes}

The primary outcome measure was the incidence of CV portrelated infectious events that required port removal within 1 year after the initial placement. The secondary outcome measures were all-cause events that required port removal. The occurrence of clinical events was determined from inpatient/ outpatient medical records. All follow-up data within 1 year after the initial placement were collected. Patients who did not experience any events or died were censored on the date of the final observation. Other relevant clinical data, including age, sex, BMI, Charlson comorbidity index (CCI), American Society of Anesthesiologists performance status (ASA-PS), lymphocyte count, serum albumin level, site of malignancy, grade of surgeon, port-insertion vessel, and the side of portinsertion, were also obtained from the electronic medical records.

\section{Statistical analyses}

Categorical data were expressed as frequencies and percentages and compared between groups using the chi-squared test. Non-normally distributed variables were presented as medians (interquartile range [IQR]) and compared between groups using the Kruskal-Wallis test. Event-free survival curves were constructed using the Kaplan-Meier method and compared using the log-rank test. Univariate and multivariate Cox proportional hazard models were used to calculate hazard ratios (HRs) and 95\% confidence intervals (CI) for the outcomes. All statistical analyses were conducted using $\mathrm{R}$ version 3.5.1 (R Foundation for Statistical Computing, Vienna, Austria). A $p$ value $<0.05$ was considered statistically significant. 


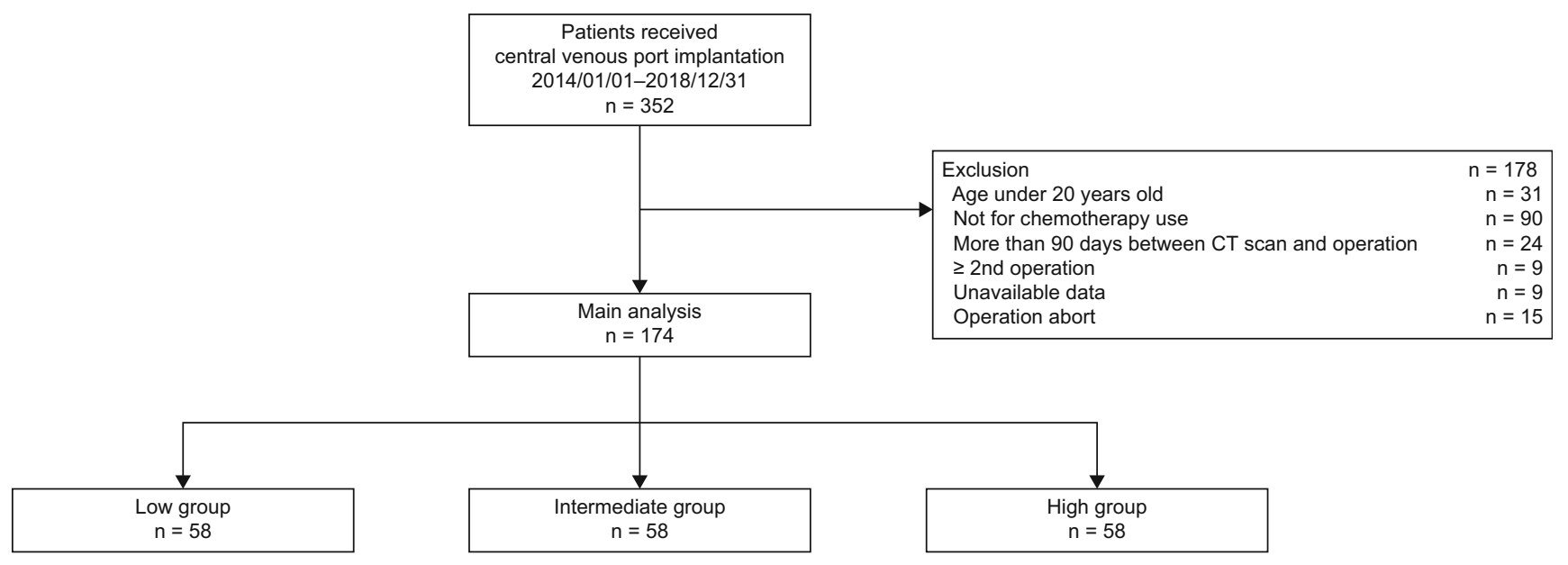

Fig. 1 Patient selection flowchart

\section{Results}

\section{Baseline patient characteristics}

Of the 352 patients who underwent $\mathrm{CV}$ port implantation, 174 were included in the analysis. In total, 58 (33.3\%) patients were assigned to each SFA group. The baseline patient characteristics according to the SFA are listed in Table 1. The median (IQR) SFA was 18.2 (6.3-29.0), 60.0 (51.2-68.9), and 118.8 $\mathrm{m}^{2}$ (93.5-150.0) in the low, intermediate, and high SFA groups, respectively. The low-SFA group included more older adults as well as more individuals with gastrointestinal cancers. Individuals in this group also had a lower BMI. There were no significant differences in other baseline characteristics, including CCI, ASA-PS, serum lymphocyte counts, serum albumin level, grade of surgeon, access side, and access site.

\section{Short- and long-term outcomes}

The short-term and long-term complications are listed in Table 2. The median follow-up period was 306 days (IQR, 167-590). During the follow-up period, 26 patients experienced the following long-term clinical events: 19 patients, infection; 2 patients, port dislocation; and 5 patients, port obstruction. The intermediate group exhibited a low rate of infection. The incidence rate of complications per 1000 catheter days was $0.51,0.19$, and 0.4 in the low, intermediate, and high SFA groups, respectively. In terms of short-term complications, such as arterial puncture, pneumothorax, or external jugular vein injury, there were no statistically significant differences among the groups.

\section{Association between SFA in the midclavicular line and long-term clinical outcomes}

The Kaplan-Meier curves for infectious and all-cause complications are shown in Fig. 3. Within a median follow-up period of 306 days, the intermediate SFA group showed a significantly higher infection-free survival (low vs. intermediate vs. high: $80.4 \%$ vs. $97.7 \%$ vs. $83.4 \%$, respectively, $p=0.034$ ). In contrast, there was no significant difference in the overall complication-free survival among the three groups (low vs. intermediate vs. high: $80.4 \%$ vs. $88.9 \%$ vs. $81.8 \%$, respectively, $p=0.29$ ).

Fig. 2 A typical image used to measure the subcutaneous fat area in the midclavicular line using multi-detector computed tomography data. Regions in blue indicate the subcutaneous fat area

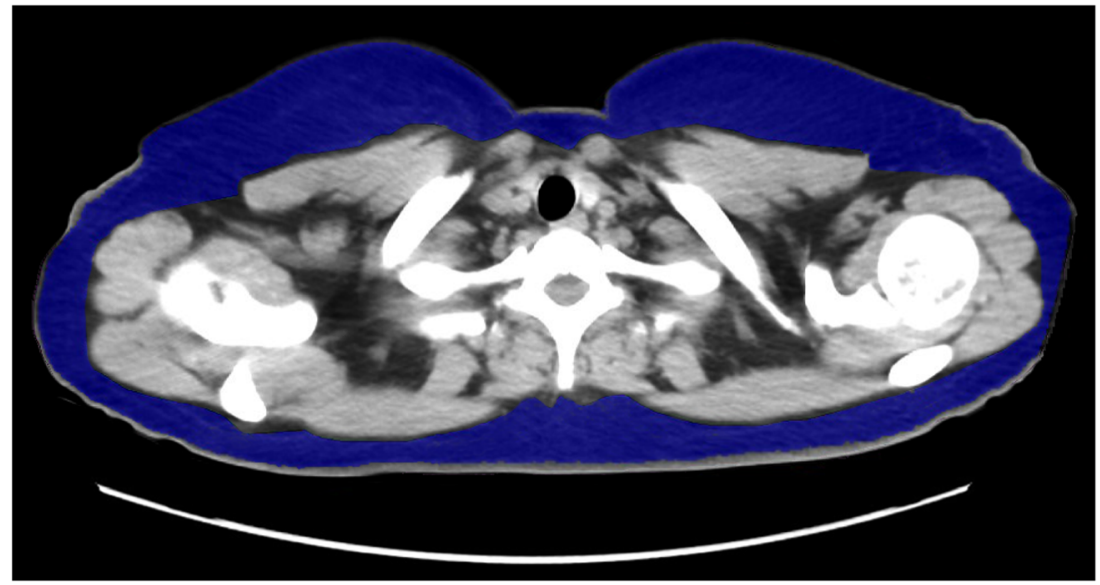


Table 1 Patients characteristics

\begin{tabular}{|c|c|c|c|c|c|c|c|c|}
\hline \multirow{3}{*}{ Age in years, $n(\%)$} & & \multirow{2}{*}{\multicolumn{2}{|c|}{$\frac{\text { Low SFA group }}{(n=58)}$}} & \multirow{2}{*}{\multicolumn{2}{|c|}{$\frac{\text { Intermediate SFA group }}{(n=58)}$}} & \multirow{2}{*}{\multicolumn{2}{|c|}{$\begin{array}{l}\text { High SFA group } \\
(n=58)\end{array}$}} & \multirow{3}{*}{$\frac{p \text { value }}{0.019}$} \\
\hline & & & & & & & & \\
\hline & & 66 & \multirow{2}{*}{$\begin{array}{l}(61.5-71.8) \\
(46.6 \%)\end{array}$} & 67 & $(59.5-72.8)$ & 62 & \multirow{2}{*}{$\begin{array}{l}(56.0-67.8) \\
(56.9 \%)\end{array}$} & \\
\hline & $<65$ & 27 & & 20 & $(34.5 \%)$ & 33 & & 0.053 \\
\hline & $\geq 65$ & 31 & $(53.4 \%)$ & 38 & $(65.5 \%)$ & 25 & $(43.1 \%)$ & \\
\hline \multicolumn{9}{|l|}{ BMI $\left(\mathrm{kg} / \mathrm{m}^{2}\right), n(\%)$} \\
\hline & $<18.5$ & 25 & $(43.1 \%)$ & 6 & $(10.3 \%)$ & 0 & $(0.0 \%)$ & $<0.001$ \\
\hline & $18.5-24.9$ & 33 & $(56.9 \%)$ & 45 & $(77.6 \%)$ & 17 & $(29.3 \%)$ & \\
\hline & $25-29.9$ & 0 & $(0.0 \%)$ & 7 & $(12.1 \%)$ & 30 & $(51.7 \%)$ & \\
\hline & $\geq 30$ & 0 & $(0.0 \%)$ & 0 & $(0.0 \%)$ & 11 & $(19.0 \%)$ & \\
\hline \multicolumn{9}{|l|}{ Sex, $n(\%)$} \\
\hline & Male & 43 & $(74.1 \%)$ & 29 & $(50.0 \%)$ & 15 & $(25.9 \%)$ & $<0.001$ \\
\hline & Female & 15 & $(25.9 \%)$ & 29 & $(50.0 \%)$ & 43 & $(74.1 \%)$ & \\
\hline \multicolumn{9}{|l|}{ CCI, $n(\%)$} \\
\hline & 0 & 29 & $(50.0 \%)$ & 19 & $(32.8 \%)$ & 22 & $(37.9 \%)$ & 0.075 \\
\hline & 1,2 & 25 & $(43.1 \%)$ & 34 & $(58.6 \%)$ & 25 & $(43.1 \%)$ & \\
\hline & $\geq 3$ & 4 & $(6.9 \%)$ & 5 & $(8.6 \%)$ & 11 & $(19.0 \%)$ & \\
\hline \multicolumn{9}{|l|}{ Performance status (ASA), $n(\%)$} \\
\hline & I & 17 & $(29.3 \%)$ & 21 & $(36.2 \%)$ & 18 & $(31.0 \%)$ & 0.56 \\
\hline & II & 22 & $(37.9 \%)$ & 24 & $(41.4 \%)$ & 28 & $(48.3 \%)$ & \\
\hline & III & 16 & $(27.6 \%)$ & 12 & $(20.7 \%)$ & 12 & $(20.7 \%)$ & \\
\hline & IV & 3 & $(5.2 \%)$ & 1 & $(1.7 \%)$ & 0 & $(0.0 \%)$ & \\
\hline \multicolumn{9}{|l|}{ Lymphocyte count $(/ \mu \mathrm{L}), n(\%)$} \\
\hline & $>1500$ & 18 & $(31.0 \%)$ & 20 & $(34.5 \%)$ & 24 & $(41.4 \%)$ & 0.50 \\
\hline & $\leq 1500$ & 40 & $(69.0 \%)$ & 38 & $(65.5 \%)$ & 34 & $(58.6 \%)$ & \\
\hline \multicolumn{9}{|l|}{ Serum albumin $(\mathrm{g} / \mathrm{dL}), n(\%)$} \\
\hline & $\geq 3.5$ & 31 & $(53.4 \%)$ & 30 & $(51.7 \%)$ & 37 & $(63.8 \%)$ & 0.37 \\
\hline & $\leq 3.4$ & 27 & $(46.6 \%)$ & 28 & $(48.3 \%)$ & 21 & $(36.2 \%)$ & \\
\hline \multicolumn{9}{|l|}{ Site of malignancy, $n(\%)$} \\
\hline & Gastrointestinal & 33 & $(56.9 \%)$ & 30 & $(51.7 \%)$ & 24 & $(41.4 \%)$ & 0.0058 \\
\hline & Pancreas & 14 & $(24.1 \%)$ & 14 & $(24.1 \%)$ & 7 & $(12.1 \%)$ & \\
\hline & Gynecologic & 5 & $(8.6 \%)$ & 3 & $(5.2 \%)$ & 7 & $(12.1 \%)$ & \\
\hline & Hematological & 2 & $(3.4 \%)$ & 8 & $(13.8 \%)$ & 5 & $(8.6 \%)$ & \\
\hline & Other & 4 & $(6.9 \%)$ & 3 & $(5.2 \%)$ & 15 & $(25.9 \%)$ & \\
\hline Grade of surgeon, median (IQR) & & 3 & $(2-3)$ & 3 & $(3-3)$ & 3 & $(3-3)$ & 0.20 \\
\hline \multicolumn{9}{|l|}{ Access side, $n(\%)$} \\
\hline & Right & 56 & $(96.6 \%)$ & 52 & $(89.7 \%)$ & 51 & $(87.9 \%)$ & 0.23 \\
\hline & Left & 2 & $(3.4 \%)$ & 6 & $(10.3 \%)$ & 7 & $(12.1 \%)$ & \\
\hline \multicolumn{9}{|l|}{ Access site, $n(\%)$} \\
\hline & Internal jugular & 53 & $(91.4 \%)$ & 55 & $(94.8 \%)$ & 58 & $(100.0 \%)$ & 0.10 \\
\hline & Subclavicular & 5 & $(8.6 \%)$ & 3 & $(5.2 \%)$ & 0 & $(0.0 \%)$ & \\
\hline Subcutaneous fat area on midclavicular line (m2) & & 18.2 & $(6.3-29.0)$ & 60.0 & $(51.2-68.9)$ & 118.8 & $(93.5-150.0)$ & $<0.001$ \\
\hline
\end{tabular}

$A S A$, American Society of Anaesthesiologists; $B M I$, body mass index; $C C$, Charlson comorbidity index; $I Q R$, interquartile range

The results of the Cox regression analysis for cumulative incidence of infectious complications are shown in Table 3. In the univariate analysis, the HRs for infectious complications were 9.00 (95\% CI: $1.14-71.04, p=0.037)$ and 9.08 (95\% CI:
$1.15-71.66, p=0.036$ ) for the low and high SFA groups, respectively. After adjusting for BMI, age, and sex, low SFA was independently associated with a higher risk of infectious complications, with an HR of 9.45 (95\% CI: 1.07-83.22, $p=0.043$ ). 
Table 2 Short- and long-term outcomes

\begin{tabular}{|c|c|c|c|c|c|c|c|}
\hline \multirow[b]{3}{*}{ Operation time (min), median (IQR) } & \multirow{2}{*}{\multicolumn{2}{|c|}{$\frac{\text { Low SFA group }}{(n=58)}$}} & \multirow{2}{*}{\multicolumn{2}{|c|}{$\begin{array}{l}\text { Intermediate SFA group } \\
(n=58)\end{array}$}} & \multirow{2}{*}{\multicolumn{2}{|c|}{$\frac{\text { High SFA group }}{(n=58)}$}} & \multirow{3}{*}{$\frac{p \text { value }}{0.14}$} \\
\hline & & & & & & & \\
\hline & \multirow{4}{*}{$\begin{array}{l}36.5 \\
0 \\
0.51\end{array}$} & $(30.3-45.0)$ & 36.5 & $(31.0-46.0)$ & 39.5 & $(34.0-49.8)$ & \\
\hline Blood loss (mL), median (IQR) & & $(0-0)$ & 0 & $(0-0)$ & 0 & $(0-0)$ & 0.16 \\
\hline Incidence rate of complications per 1000 catheter days & & & 0.19 & & 0.4 & & \\
\hline \multicolumn{7}{|l|}{ Complications, $n(\%)$} & \\
\hline All complications & 10 & $(17.2 \%)$ & 5 & $(8.6 \%)$ & 11 & $(19.0 \%)$ & 0.25 \\
\hline Dislocation & 1 & $(1.7 \%)$ & 1 & $(1.7 \%)$ & 0 & $(0.0 \%)$ & 1.0 \\
\hline Infection & 9 & $(15.5 \%)$ & 1 & $(1.7 \%)$ & 9 & $(15.5 \%)$ & 0.023 \\
\hline Obstruction & 0 & $(0.0 \%)$ & 3 & $(5.2 \%)$ & 2 & $(3.4 \%)$ & 0.37 \\
\hline \multicolumn{8}{|l|}{ Short-term complications, $n(\%)$} \\
\hline Yes & 1 & $(1.7 \%)$ & 2 & $(3.4 \%)$ & 0 & $(0.0 \%)$ & 0.77 \\
\hline Artery puncture & 1 & $(1.7 \%)$ & 0 & $(0.0 \%)$ & 0 & $(0.0 \%)$ & 1.0 \\
\hline Pneumothorax & 0 & $(0.0 \%)$ & 1 & $(1.7 \%)$ & 0 & $(0.0 \%)$ & 1.0 \\
\hline External jugular vein injury & 0 & $(0.0 \%)$ & 1 & $(1.7 \%)$ & 0 & $(0.0 \%)$ & 1.0 \\
\hline
\end{tabular}

$I Q R$, interquartile range

\section{Discussion}

To date, there has been no evidence to support the association between SFA and CV port-related infections. This study showed that low SFA in the midclavicular line was significantly associated with a high incidence of infectious portrelated complications within 1 year in patients who underwent CV port-insertion for chemotherapy. Evidence to date has been limited or sparse in this field. These findings are clinically significant as they substantiate the surgeons' impression that patients who have a low fat area in the surgical site may have a high risk of infections.

Preoperative evaluation of a patient's risk of infectious complications according to SFA will be helpful to prevent port-related complications, ensure compliance with the chemotherapy plan, and maintain a sufficient dose intensity. This study showed that low, but not high, SFA in the midclavicular

(a)

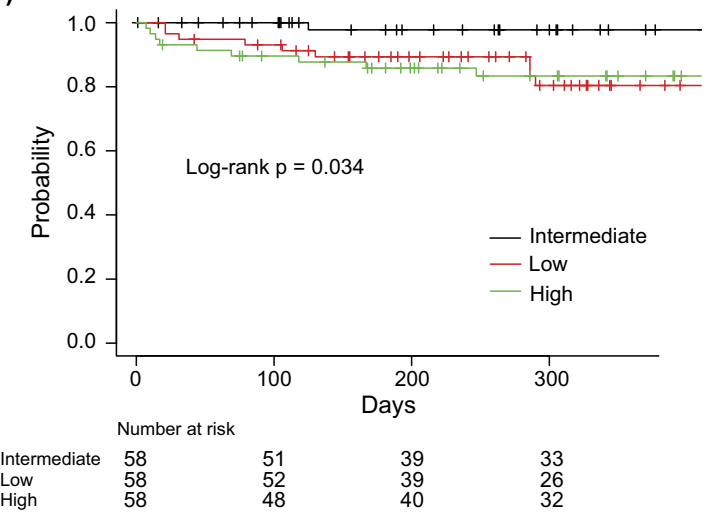

line was significantly associated with a high incidence of infectious port-related complications in patients who underwent CV port-insertion for chemotherapy. Previous studies have reported the relationship between BMI and infectious events; however, these reports maintained that both low and high BMI had an impact on the risk of infections [5, 6]. This discrepancy in the results may be because BMI is a nonspecific parameter that cannot differentiate between fat mass and body lean mass or between visceral fat mass and subcutaneous fat mass. Meanwhile, the SFA in the midclavicular line is a more specific parameter than BMI for evaluating the volume of SFA at the surgical site.

In this study, the SFA at the level of the midclavicular line was utilized to semi-automatically measure the midclavicular level fat area in the surgical site. Low SFA in the midclavicular line was found to be an independent risk factor for port-related infectious events, after adjusting for BMI, in

(b)

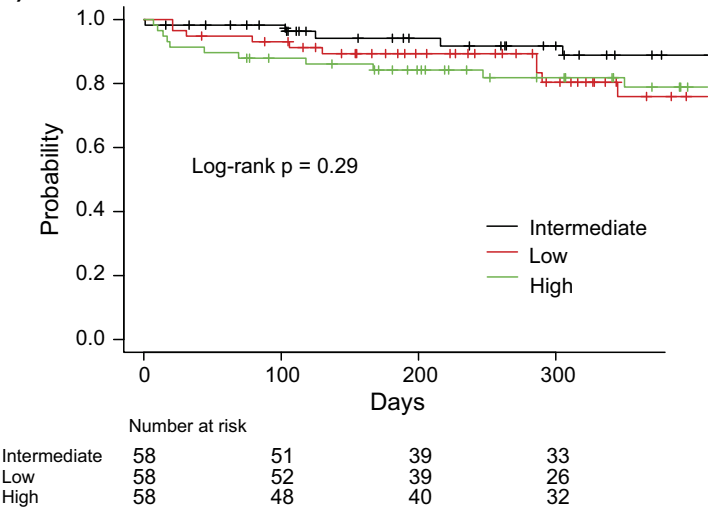

Fig. 3 Kaplan-Meier analysis of a infectious complication events and b all complication events according to the amount of subcutaneous fat area 
Table 3 Multivariate Cox proportional hazard model for infectious complications

\begin{tabular}{|c|c|c|c|c|c|c|}
\hline \multirow[t]{2}{*}{ Variables } & \multicolumn{3}{|l|}{ Unadjusted } & \multicolumn{3}{|l|}{ Adjusted } \\
\hline & HR & $95 \% \mathrm{CI}$ & $p$ value & HR & $95 \% \mathrm{CI}$ & $p$ value \\
\hline \multicolumn{7}{|c|}{ Subcutaneous fat area on midclavicular } \\
\hline Intermediate group & (Reference) & & & (Reference) & & \\
\hline Low group & 9.00 & $(1.14-71.04)$ & 0.037 & 9.45 & $(1.07-83.22)$ & 0.043 \\
\hline High group & 9.08 & $(1.15-71.66)$ & 0.036 & 4.96 & $(0.47-52.06)$ & 0.18 \\
\hline \multicolumn{7}{|l|}{ BMI } \\
\hline$<18.5$ & (Reference) & & & (Reference) & & \\
\hline $18.5-24.9$ & 0.81 & $(0.22-3.08)$ & 0.76 & 1.24 & $(0.30-5.06)$ & 0.77 \\
\hline $25-29.9$ & 1.35 & $(0.32-5.65)$ & 0.68 & 2.50 & $(0.25-24.82)$ & 0.44 \\
\hline$\geq 30$ & 2.87 & $(0.58-14.24)$ & 0.20 & 4.76 & $(0.42-54.40)$ & 0.21 \\
\hline \multicolumn{7}{|l|}{ Age } \\
\hline$<65$ & (Reference) & & & (Reference) & & \\
\hline$\geq 65$ & 0.49 & $(0.19-1.24)$ & 0.13 & 0.65 & $(0.25-1.69)$ & 0.37 \\
\hline \multicolumn{7}{|l|}{ Sex } \\
\hline Male & (Reference) & & & (Reference) & & \\
\hline Female & 0.72 & $(0.29-1.79)$ & 0.48 & 0.68 & $(0.24-1.95)$ & 0.48 \\
\hline
\end{tabular}

Adjusted for BMI, age, sex

$B M I$, body mass index; $C I$, confidence interval; $H R$, hazard ratio patients who underwent CV port-insertion for chemotherapy. These findings indicate that SFA in the midclavicular line is a more appropriate measure than BMI. Importantly, SFA could be a simple and useful risk indicator in patients undergoing an indwelling port procedure for chemotherapy.

SFA in the current study was measured using CT scans, which is the gold standard for evaluating cancer progression. SFA can also be measured using semi-automated methods for the assessment of body composition [3]. Therefore, SFA can be easily measured preoperatively using various modalities, including CT [7]. Previous studies have reported several risk factors for port-related infections, including hematological malignancies, pancreatic cancer, head and neck cancer, neutropenia, chronic steroid use, lack of perioperative antibiotic use, poor performance status, previous infection, parenteral nutrition, immediate palliative care after implantation, chemotherapy in the non-adjuvant setting, and surgery conducted by an inexperienced surgeon [8-12]. Some previous studies also evaluated the clinical relevance between surgical site infection and its morphometric characteristics, other than BMI, in abdominal surgeries [4, 13-15]. They suggested that a high amount of SFA increased the risk of surgical site infections. Another study indicated a relationship between low BMI and $\mathrm{CV}$ port complications [16]. However, these studies did not focus on the location of $\mathrm{CV}$ port insertion or conduct a morphometric assessment; our research focused on both topics.

Low SFA in the implantation site may not only be a risk factor for exposure of the $\mathrm{CV}$ port, but also suggests undernutrition, which increases the susceptibility to infection [17] and port-related complications.

This study has some limitations. First, the sample size was relatively small, which may have caused an inadequate adjustment for confounding factors and wide confidential intervals. However, we were able to adjust for the important confounders (BMI, age, sex) in the analysis of the relationship between SFA and infectious events. Additionally, we showed a significant correlation between SFA and infectious events. Second, the SFA calculation method was originally developed for measuring fat areas at the level of the umbilicus, not at the level of the midclavicular line. Therefore, the results should be interpreted cautiously until confirmed by further studies. Third, it is possible for cancer patients to show significant changes in body shape within 90 days. This can be a source of measurement bias.

However, this study provided the new finding that low SFA in the midclavicular line is significantly associated with a high incidence of infectious port-related complications in patients who underwent $\mathrm{CV}$ port-insertion for chemotherapy. These findings verified the surgeons' belief that patients with low SFA have a high risk of infectious events. SFA can be conveniently measured in cancer patients using CT, which is routinely performed in this context. Further, SFA is a more specific indicator than BMI. Therefore, despite this study's limitations, the present findings provide new insight into the risk assessment of $\mathrm{CV}$ port-related infections. Our research suggests that physicians should personalize the choice of 
vascular access to meet the patients' treatment regimen necessities, while also seeking to reduce the risk of infection and other port-related complications in patients with low SFA. Currently, arm ports have been developed as a new approach to indwelling infusion systems. These devices are smaller and show no greater risk of harm compared with ports placed in the chest [18]. In order to reduce the risk of infection, the port indwelling site might be changed from the chest to the arm in adult patients with lower SFAs. Further studies are needed to validate the results and determine the generalizability of our findings in other populations.

\section{Conclusion}

CT-derived SFA in the midclavicular line is an independent indicator of infectious complications in chemotherapy settings, making it a useful indicator when choosing other types of vascular access for chemotherapy administration, such as an arm port, and optimizing the patient's nutritional status.

Availability of data and material Data supporting the findings of this study are available from the corresponding author (J.S.) upon reasonable request.

Code availability Raw data were generated at the Toyohashi Municipal Hospital. Derived data supporting the findings of this study are available from the corresponding author on request.

Author contribution J.S. and H.K. designed the project and performed the measurement. M.H., K.H., and T.K. planned and supervised the work. J.S. and H.K. processed the experimental data, performed the analysis, drafted the manuscript, and created the figures. Y.S., T.A., M.F., A.A., A.I., K.I., K.O., M.A., T.A., H.G., S.A., T.H., and K.K. helped to interpret the results and prepare the manuscript. All authors have read and approved the final version of the manuscript.

\section{Declarations}

Ethics approval Any accompanying images and procedures were obtained and conducted following the Declaration of Helsinki. The study protocol was approved by the Institutional Review Board of Toyohashi Municipal Hospital (July $3^{\text {rd }}, 2019$ / No. 451)

Consent to participate Patients participated through an opt-out methodology.

Consent for publication Consent for publication was obtained through an opt-out methodology. Institutional Review Board approved the publication of our manuscript.

Competing interests The authors declare no competing interests.

Open Access This article is licensed under a Creative Commons Attribution 4.0 International License, which permits use, sharing, adaptation, distribution and reproduction in any medium or format, as long as you give appropriate credit to the original author(s) and the source, provide a link to the Creative Commons licence, and indicate if changes were made. The images or other third party material in this article are included in the article's Creative Commons licence, unless indicated otherwise in a credit line to the material. If material is not included in the article's Creative Commons licence and your intended use is not permitted by statutory regulation or exceeds the permitted use, you will need to obtain permission directly from the copyright holder. To view a copy of this licence, visit http://creativecommons.org/licenses/by/4.0/.

\section{References}

1. Yagi T, Sakamoto T, Nakai K, Tanizawa M, Okabe T, Hoshikawa N, Kohatada M, Kitagawa F, Hanya R, Kotani M (2016) A questionnaire-based assessment of the anxiety, satisfaction and discomfort experienced by Japanese cancer patients during the use of central venous ports. Intern Med 55:2393-2399. https://doi.org/10. 2169/internalmedicine.55.6032

2. Carratalà J, Niubó J, Fernández-Sevilla A, Juvé E, Castellsagué X, Berlanga J, Liñares J, Gudiol F (1999) Randomized, double-blind trial of an antibiotic-lock technique for prevention of gram-positive central venous catheter-related infection in neutropenic patients with cancer. Antimicrob Agents Chemother 43:220-2204. https:// doi.org/10.1128/aac.43.9.2200

3. Inoue M, Sobue T, Tsugane S, JPHC Study Group (2004) Impact of body mass index on the risk of total cancer incidence and mortality among middle-aged Japanese: data from a large-scale populationbased cohort study-the JPHC study. Cancer Causes Control 15: 671-680. https://doi.org/10.1023/b:caco.0000036177.77953.47

4. Lee JS, Terjimanian MN, Tishberg LM, Alawieh AZ, Harbaugh CM, Sheetz KH, Holcombe SA, Wang SC, Sonnenday CJ, Englesbe MJ (2011) Surgical site infection and analytic morphometric assessment of body composition in patients undergoing midline laparotomy. J Am Coll Surg 213:236-244. https://doi.org/10. 1016/j.jamcollsurg.2011.04.008

5. Harpsøe MC, Nielsen NM, Friis-Møller N, Andersson M, Wohlfahrt J, Linneberg A, Nohr EA, Jess T (2016) Body mass index and risk of infections among women in the Danish national birth cohort. Am J Epidemiol 183:1008-1017. https://doi.org/10. 1093/aje/kwv300

6. Atamna A, Elis A, Gilady E, Gitter-Azulay L, Bishara J (2017) How obesity impacts outcomes of infectious diseases. Eur J Clin Microbiol Infect Dis 36:585-591. https://doi.org/10.1007/s10096016-2835-1

7. Yoshizumi T, Nakamura T, Yamane M, Waliul Islam AH, Menju M, Yamasaki K, Arai T, Kotani K, Funahashi T, Yamashita S, Matsuzawa Y (1999) Abdominal fat: standardized technique for measurement at CT. Radiology 211:283-286. https://doi.org/10. 1148/radiology.211.1.r99ap15283

8. Chang YF, Lo AC, Tsai CH, Lee PY, Lee PY, Sun S, Chang TH, Chen CC, Chang YS, Chen JR (2013) Higher complication risk of totally implantable venous access port systems in patients with advanced cancer - a single institution retrospective analysis. Palliat Med 27:185-191. https://doi.org/10.1177/0269216311428777

9. Chen IC, Hsu C, Chen YC, Chien SF, Kao HF, Chang SY, Hu FC, Yeh KH (2013) Predictors of blood stream infection associated with permanently implantable venous port in solid cancer patients. Ann Oncol 24:463-468

10. Toure A, Vanhems P, Lombard-Bohas C, Cassier P, Péré-Vergé D, Souquet JC, Ecochard R, Chambrier C (2012) Totally implantable central venous access port infections in patients with digestive cancer incidence and risk factors. Am J Infect Control 40:935-939. https://doi.org/10.1016/j.ajic.2012.01.024 
11. Hsieh CC, Weng HH, Huang WS, Wang WK, Kao CL, Lu MS, Wang CS (2009) Analysis of risk factors for central venous port failure in cancer patients. World J Gastroenterol 15:4709-4714

12. Wang TY, Lee KD, Chen PT, Chen MC, Chen YY, Huang CE, Kuan FC, Chen CC, Lu CH (2015) Incidence and risk factors for central venous access port-related infection in Chinese cancer patients. J Formos Med Assoc 114:1055-1060. https://doi.org/10. 1016/j.jfma.2015.06.013

13. Cai X, Shen W, Guo Z, Li Y, Cao L, Gong J, Zhu W (2018) Thickness of subcutaneous fat is a predictive factor of incisional surgical site infection in Crohn's disease surgery: a retrospective study. Gastroenterology Res Pract 2018:1546075

14. Nakagawa H, Ohno K, Ikeda S, Muto M (2016) The effect of preoperative subcutaneous fat thickness on surgical site infection risk in patients undergoing colorectal surgery: results of a multisite, prospective cohort study. Ostomy Wound Manage 62:14-20
15. Fujii T, Tsutsumi S, Matsumoto A, Fukasawa T, Tabe Y, Yajima R, Asao T, Kuwano H (2010) Thickness of subcutaneous fat as a strong risk factor for wound infections in elective colorectal surgery: impact of prediction using preoperative CT. Dig Surg 27:331335. https://doi.org/10.1159/000297521

16. Johnson JR (1995) Complications and failures of subclavian-vein catheterization. N Engl J Med 332:1579-1581

17. Mainous MR, Deitch EA (1994) Nutrition and infection. Surg Clin North Am 74:659-676

18. Li G, Zhang Y, Ma H, Zheng J (2019) Arm port vs chest port: a systematic review and meta-analysis. Cancer Manag Res 11:6099 6112. https://doi.org/10.2147/CMAR.S205988

Publisher's note Springer Nature remains neutral with regard to jurisdictional claims in published maps and institutional affiliations. 